\title{
Cardiac Arrhythmia and Injury Induced in Rats by Burst and Pulsed Mode Ultrasound With a Gas Body Contrast Agent
}

\author{
Douglas L. Miller, PhD, Chunyan Dou, MD, Benedict R. Lucchesi, MD
}

\begin{abstract}
Objective. Premature complexes (PCS) in the electrocardiogram (ECG) signal have been reported for myocardial contrast echocardiography and also for burst mode (physical therapy) ultrasound with gas body contrast agents at lower peak rarefactional pressure amplitudes (PRPAs). For contrast echocardiography, irreversibly injured cardiomyocytes have been associated with the arrhythmia. The objective was to determine whether cardiomyocyte injury is associated with the PCs induced by the burst mode at lower PRPAs. Methods. Anesthetized rats were exposed to focused 1.5-MHz ultrasound in a water bath. Evans blue dye was injected intraperitoneally to stain injured cardiomyocytes, and a perflutren lipid microsphere ultrasound contrast agent was infused intravenously. The continuous burst mode simulated physical therapy ultrasound. Intermittent 2-millisecond bursts, or envelopes of pulses simulating diagnostic ultrasound, were triggered 1:4 at end systole. Premature complexes were observed on ECG recordings, and stained cardiomyocytes were counted in frozen sections. Results. The continuous burst mode produced variable PCs and stained cells above a 0.3-MPa PRPA. The triggered bursts above $0.3 \mathrm{MPa}$ and pulse envelopes above $1.2 \mathrm{MPa}$ produced statistically significant $(P<.01) \mathrm{PCs}$ and stained cardiomyocytes. Conclusions. Irreversible cardiomyocyte injury was associated with the development of PCs for the burst mode and occurred at substantially lower PRPAs than for pulsed ultrasound. Key words: arrhythmia; contrast agent adverse effects; gas body activation; myocardial contrast echocardiography; ultrasonic cavitation biology.
\end{abstract}

\begin{abstract}
Abbreviations
ECG, electrocardiogram; PC, premature complex; PRPA, peak rarefactional pressure amplitude; PVC, premature ventricular complex; SC, stained cell; SPC, supraventricular premature complex
\end{abstract}

Received March 23, 2009, from the Departments of Radiology (D.L.M., C.D.) and Pharmacology (B.R.L.), University of Michigan, Ann Arbor, Michigan USA. Revision requested April 16, 2009. Revised manuscript accepted for publication April 26, 2009.

This work was supported by Public Health Service grant EB00338, awarded by the National Institutes of Health, US Department of Health and Human Services.

Address correspondence to Douglas L. Miller, PhD, University of Michigan Health System, 3240A Medical Sciences Building I, 1301 Catherine St, Ann Arbor, MI 48109-5667 USA.

E-mail: douglm@umich.edu

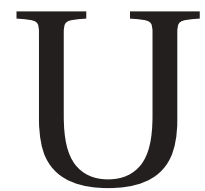

ltrasound contrast agents are approved for use in enhancing diagnostic ultrasound images. The agents consist of suspensions of gas bodies (stabilized microbubbles), which circulate in the blood pool and return strong echoes relative to the weak echoes from blood. The first application of gas body contrast agents was for echocardiography with approval to opacify the blood pool within the left ventricular chamber and to improve the delineation of the endocardial border. Myocardial contrast echocardiography is another application, which seeks to image perfusion in the cardiac muscle. This can be accomplished at relatively high pulse peak rarefactional pressure amplitudes (PRPAs) of diagnostic ultrasound by destroying the gas bodies and then observing the rate of refill of blood with fresh gas bodies into the tissue. Safety considerations for contrast enhanced diagnostic ultrasound have received substantial research interest because the interaction between ultrasound pulses and gas bodies is an impor- 
tant mechanism for nonthermal bioeffects of ultrasound. This research has led to a variety of bioeffects findings and to potential therapeutic applications. ${ }^{1}$

An intriguing bioeffect of myocardial contrast echocardiography is cardiac arrhythmia. This effect has been reported in humans ${ }^{2,3}$ as well as laboratory animals, including rats ${ }^{4}$ and dogs. ${ }^{5}$ The arrhythmia effect of contrast echocardiography occurs at relatively high PRPAs, which are also associated with gas body destabilization and destruction by the ultrasound pulses. The arrhythmia shows as premature complexes (PCs) in the electrocardiogram (ECG) signal, which occur coincidentally with the ultrasound image exposure and cease after the ultrasound is stopped. A typical exposure that elicits this arrhythmia in rats involves infusion of a contrast agent, intermittent imaging every fourth complex (1:4) at end systole, and a PRPA above about $1 \mathrm{MPa}$ at $1.7 \mathrm{MHz} .{ }^{4}$ The intermittent image exposure allows for the refill of tissue capillaries with blood containing gas bodies, which are destroyed by each image exposure. The induction of arrhythmia with a contrast agent in the circulation also has been observed with lowpower burst mode ultrasound, such as that used for physical therapy. ${ }^{3}$ Continuous burst mode (2millisecond bursts repeated at 10-millisecond intervals) exposure at $1 \mathrm{MHz}$ and 0.33 to $0.39 \mathrm{MPa}$ produced more arrhythmia than intermittent (1:4) 1.7-MHz diagnostic ultrasound at 1.0 MPa.

Another important bioeffect at relatively high PRPAs is irreversible cardiomyocyte injury. Histologic evidence of cardiomyocyte contraction band necrosis immediately after exposure has been shown for diagnostic ultrasound with a commercial contrast agent in rats. ${ }^{6}$ Irreversible cardiomyocyte injury also was identified by staining with Evans blue dye, which was detected the day after exposure. ${ }^{7}$ The incidence of the Evans blue staining was associated with the appearance of PCs in the ECG signal used for triggering images, suggesting that the 2 effects were related. Although the mean numbers of PCs and stained cardiomyocytes followed a very similar exposure-response trend with the PRPA, there was little correlation between the 2 bioeffects for separate observations in individual animals. This association was also found for high PRPAs (above the range of diagnostic ultrasound) ${ }^{8}$ Premature complexes were seen for 10 of 20 rats exposed to 3.1-MHz ultrasound with 1.3-microsecond pulses at 15.9 MPa and a perflutren protein microsphere contrast agent (Optison; GE Healthcare, Milwaukee, WI) in the circulation. Myocardial degeneration was identified by histologic staining in 16 rats, which implies that the presence of myocardial degeneration alone may not be a sufficient explanation for the PCs.

The association of arrhythmia and cardiomyocyte injury at moderate and high PRPAs suggests that the ultrasonic activation of the gas bodies nucleates cavitation, which induces both bioeffects. Cavitation is the most effective mechanism for nonthermal bioeffects of ultrasound. Cavitation nucleation by ultrasound contrast agents is important in regard to the bioeffects potential in vivo because there normally are few, if any, cavitation nuclei in the body suitable for direct activation by diagnostic ultrasound pulses. ${ }^{9}$ Ultrasound contrast agents can supply such nuclei. For example, direct evidence of cavitational activity has been obtained by detection of broadband noise emissions from the myocardium during contrast echocardiography. ${ }^{10}$ However, this picture is confused by the observation of PCs with lower-amplitude physical therapy ultrasound. ${ }^{3}$ The objective of this study was to explore the association between arrhythmia and cardiomyocyte injury by comparing these effects for continuous burst mode ultrasound, triggered single bursts, and square or ramped envelopes of ultrasound pulses (simulating diagnostic ultrasound imaging). A laboratory exposure system was developed to produce all 3 modes of exposure under similar conditions (ie, with the same 1.5$\mathrm{MHz}$ transducer) over a range of PRPAs. Premature complexes were detected in ECG recordings, and cardiomyocyte injury was detected by the Evans blue method. The results strengthen the association between the 2 bioeffects of cardiac exposure to ultrasound with gas body contrast agents.

\section{Materials and Methods}

\section{Animal Preparation}

All in vivo animal procedures were conducted with the approval and guidance of the University Committee on Use and Care of Animals. CD hairless rats (Charles River Laboratories, Wilmington, MA) were anesthetized by intraperitoneal injec- 
tion of a mixture of ketamine $(87 \mathrm{mg} / \mathrm{mL})$ and xylazine $(13 \mathrm{~mL} / \mathrm{kg})$. A 24-gauge cannula was inserted into a tail vein for intravenous injections. Evans blue dye in saline $(20 \mathrm{mg} / \mathrm{mL})$ at a dose of 100 $\mathrm{mg} / \mathrm{kg}$ was injected intraperitoneally as a vital stain for cardiomyocytes. The rats then were mounted on a holding board, and ECG needle electrodes were placed in the forelegs and right hind leg. The holding board was then mounted in a $37^{\circ} \mathrm{C}$ degassed water bath for ultrasound exposures.

The ECG signal was amplified (ECGA amplifier; Hugo Sachs Electronik, March, Germany) and sent to an oscilloscope (TDS 520B; Tektronix, Inc, Beaverton OR) and a digitizer (Powerlab 4/30; ADInstruments, Inc Colorado Springs, CO). The digitized ECG was analyzed with the aid of software (Chart Pro 5, version 5.5.5; ADInstruments, Inc), which provided automated collection of data on the heart rate and numbers of normal complexes. The software also partially automated detection of PCs. However, visual examination of the PCs was performed to classify these into 2 categories. ${ }^{11}$ Supraventricular premature complexes (SPC) including a $\mathrm{P}$ wave were seen with or without a compensatory pause. Premature ventricular complexes (PVCs) without a $\mathrm{P}$ wave but always with a compensatory pause were seen singly or followed by reentry complexes.

Definity (Lantheus Medical Imaging, Inc, North Billerica, MA), a perflutren lipid microsphere ultrasound contrast agent, was prepared fresh each day. For infusion, the agent first was diluted 50:1 in sterile saline in a 3-mL syringe, which was then used to fill a $30-\mathrm{cm}$ extension tube for connection to the tail vein cannula. The syringe was mounted in a syringe pump (11 Plus; Harvard Apparatus, Holliston MA), and the infusion rate was set to $500 \mu \mathrm{L} / \mathrm{kg} / \mathrm{min}$ for the diluted agent (10 $\mu \mathrm{L} / \mathrm{kg} / \mathrm{min}$ undiluted). Infusions with exposure were 5 minutes in duration. The total agent dose of $50 \mu \mathrm{L} / \mathrm{kg}$ was about 2.5 times the recommended (package insert) human infusion dose for diagnostic imaging.

\section{Ultrasound Exposure}

Ultrasound exposure was provided by a laboratory system with guidance by diagnostic ultrasound imaging, as described previously. ${ }^{12}$ The laboratory exposure system consisted of a transducer, power amplifier (A-500; Electronic
Navigation Industries, Rochester, NY), function generator for generating a pulse train (3314A; Hewlett-Packard Company, Palo Alto, CA), and an arbitrary waveform generator (33220A; Agilent Technologies, Loveland, CO). The function generator was set to provide a $1.5-\mathrm{MHz}$ continuous signal or a pulse train with 3 cycle pulses and a 230-microsecond pulse repetition period. The arbitrary waveform generator was used for amplitude modulation of the continuous or pulsed signals. Four different signals were produced: continuous burst ( 2 milliseconds on, 6 milliseconds off), triggered single bursts (2 milliseconds), triggered pulse envelope (2millisecond square envelope), and triggered ramped pulse envelopes (50-millisecond envelope ramping either up or down). The continuous burst simulated the therapeutic ultrasound mode commonly used for physical therapy. The triggering was used to allow intermittent exposure timed from the ECG. The triggered pulse envelope was used to partly simulate intermittent diagnostic ultrasound imaging. Finally, the ramp-modulated pulses were used to induce or suppress gas body activity within the tissue, as described previously. ${ }^{12}$ Triggering was set at end systole from the ECG signal displayed on the oscilloscope by the delayed trigger function. The oscilloscope delayed trigger out signal was used to externally trigger the arbitrary waveform generator to generate the amplitude modulation envelopes for the function generator. In addition, the modulation signal was digitized, and the number of triggers during exposure was obtained with the Chart Pro software.

A damped single-element transducer (Panametrics A3464, Olympus NDT, Inc, Waltham, MA) with a 1.9- $\mathrm{cm}$ diameter and a 3.8-cm focus was used for ultrasound exposure. The PRPA of each signal was measured with a calibrated hydrophone (805; Sonora Medical Systems, Inc, Longmont, CO). The 3-cycle pulse signals produced 1.8-microsecond ultrasound pulses. A diagnostic ultrasound machine (VingMed System 5; GE Healthcare, Cincinnati, $\mathrm{OH}$ ) was used to image the heart with an $8-\mathrm{MHz}$ probe before exposure. Once an image of the rat heart that showed a clear path to the heart was obtained, the exposure transducer was moved into the same position to aim at the left ventricle. 
For evaluation of Evans blue staining of cardiomyocytes, the hearts were removed the day after exposure and cleared of most blood with heparin and saline. The heart was trimmed and then frozen in embedding medium (Tissue-Tek O.C.T. compound; Sakura Finetek USA, Inc, Torrance, CA) on dry ice. These samples were stored in $\mathrm{a}-80^{\circ} \mathrm{C}$ freezer for later sectioning on a frozen section microtome. To search for any stained cardiomyocytes, 10 - $\mu \mathrm{m}$ sections were cut at each $200 \mu \mathrm{m}$ into the sample, which typically gave 20 to 24 slides from the exposed central portion of the heart. Each slide was examined with a microscope using fluorescence illumination to estimate the number of Evans blue-stained (red fluorescent) cells. The slide with the most cell staining was used to obtain a detailed count of the number of stained cells (SCs) over the entire section.

Figure 1. Examples of PCs seen with contrast-enhanced ultrasound exposures in 1-second segments of ECG data (arbitrary 0 time start): a, SPC (arrow) without a compensatory pause; b, SPC (arrow) with a compensatory pause after the complex; c, PVC with positive excursion and a compensatory pause; d, PVC with negative excursion and a compensatory pause; e, PVC followed by reentry and a compensatory pause. The continuous burst mode at $0.5 \mathrm{MPa}$ was used for $\mathbf{a}$ and $\mathbf{b}$. For $\mathbf{c}-\mathbf{e}$, the triggered single burst mode was used, with the exposure time indicated by the vertical bars.

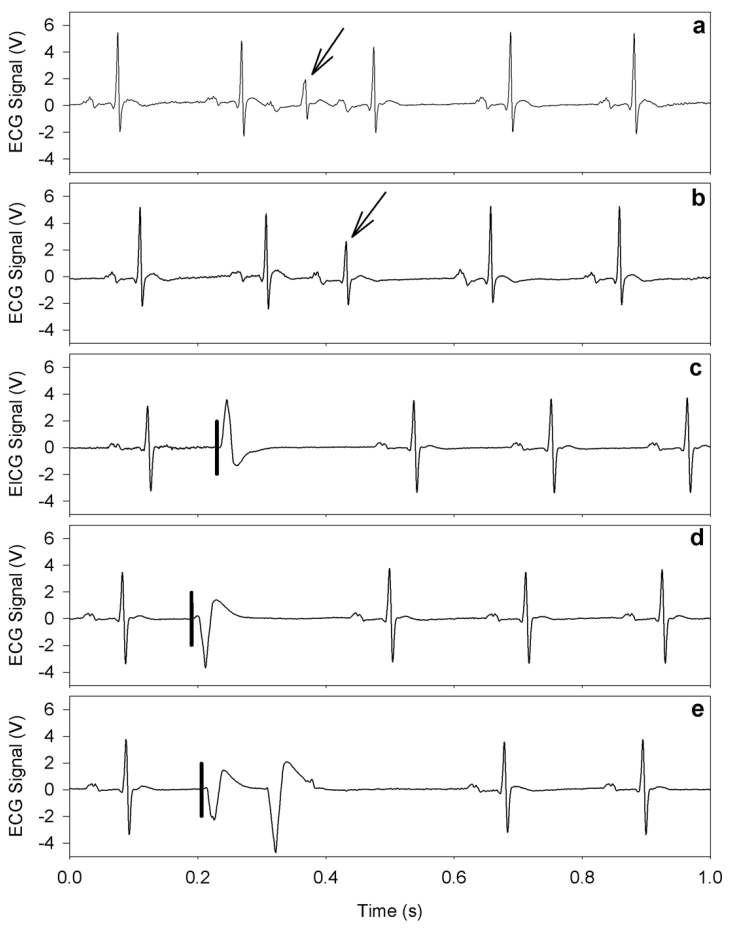

The study was conducted in 16 groups of 5 rats each and 1 group of 4 rats. For the continuous burst mode, 1 sham group was exposed to $1 \mathrm{MPa}$, and 5 groups were exposed to different PRPAs in the range of 0.25 to $1.0 \mathrm{MPa}$ (in 3-dB steps). For the triggered single burst mode, 6 groups were exposed to PRPAs in the range of 0.25 to $1.4 \mathrm{MPa}$. For the triggered pulse envelope, 4 groups were exposed to PRPAs in the range of 1.0 to $2.8 \mathrm{MPa}$. Two groups were exposed with the ramped pulse envelopes with a maximum $2.8 \mathrm{MPa}, 1$ with the ramp up (pulse PRPA increasing with time) and 1 with the ramp down (pulse PRPA decreasing with time). For all groups, the ECG was monitored and recorded for 1 minute to confirm stable heart function under anesthesia, and no PCs were observed during this preexposure period. For exposure, the infusion was started; the ultrasound was switched on; and the ECG was recorded for a manually timed period of 5 minutes. The ultrasound and infusion were then switched off. The ECG was monitored and recorded for an additional 5 minutes, during which no PCs were seen (ie, any ultrasound-induced arrhythmia ceased with the cessation of ultrasound exposure). Results are reported as mean \pm 1 SD or plotted with SE bars. For statistical analysis, the Student $t$ test and Mann-Whitney rank sum test, as appropriate, were used to compare means of the measured parameters, with statistical significance assumed at $P<.05$.

\section{Results}

The different types of PCs are shown in Figure 1. The number of PCs are shown in Figure 2 for 1.0$\mathrm{MPa}$ continuous burst and triggered single burst exposures. The SPCs were primarily seen in the continuous burst mode with none occurring in the triggered burst mode. Premature ventricular complexes were the predominant arrhythmias for the triggered single burst and triggered pulse envelope exposures. PVCs had both positive and negative excursions of varying magnitudes. The PCs began at the point of the triggered exposure with a delay of about 15 to 20 milliseconds to the peak of the complex.

The exposure responses of the 2 bioeffects are shown in Figure 3 for the continuous burst, Figure 4 for the triggered single bursts, and Figure 5 for 


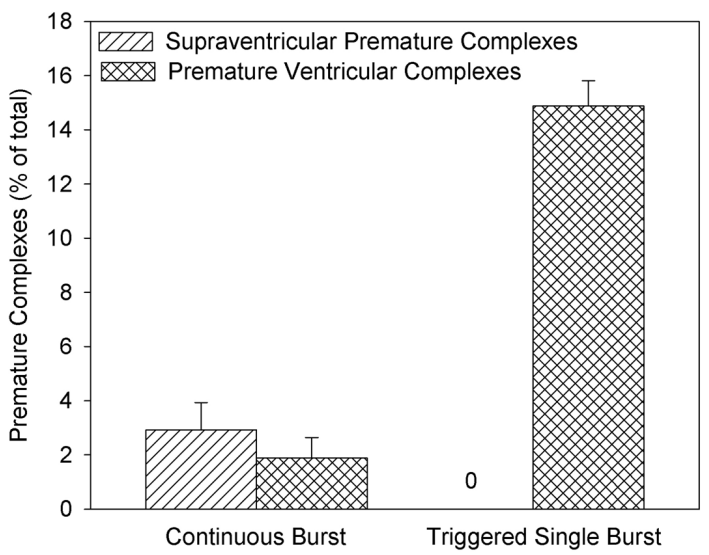

Figure 2. Numbers of PCs resembling Figure 1, a and $\mathbf{b}$, and PVCs resembling Figure 1, c-e, for the continuous burst and triggered single burst modes at $1.0 \mathrm{MPa}$. Premature ventricular complexes were the predominant PCs in the triggered mode.

the triggered pulse envelope. For all 3 modes, both effects were essentially 0 and then rose steadily at higher PRPAs. For the continuous burst mode, the SC count was small and variable, with SCs observed in 3 of 5 rat heart samples at $0.35 \mathrm{MPa}, 2$ of 5 at $0.5 \mathrm{MPa}$, and 3 of 4 at $0.7 \mathrm{MPa}$. The count achieved statistical significance only at the 1.0-MPa level. However, the PCs were observed for all rats exposed at and above 0.35 $\mathrm{MPa}$. The PCs were statistically significant at $0.35 \mathrm{MPa}$, which indicates a threshold at $0.3 \mathrm{MPa}$ (midway between the lowest level with a significant effect and the highest level without a significant effect). The exposures triggered from the

Figure 3. Means with SE bars for the numbers of PCS and Evans blue-stained SCS found for the continuous burst mode. The data trends with PRPAs are similar for both effects.

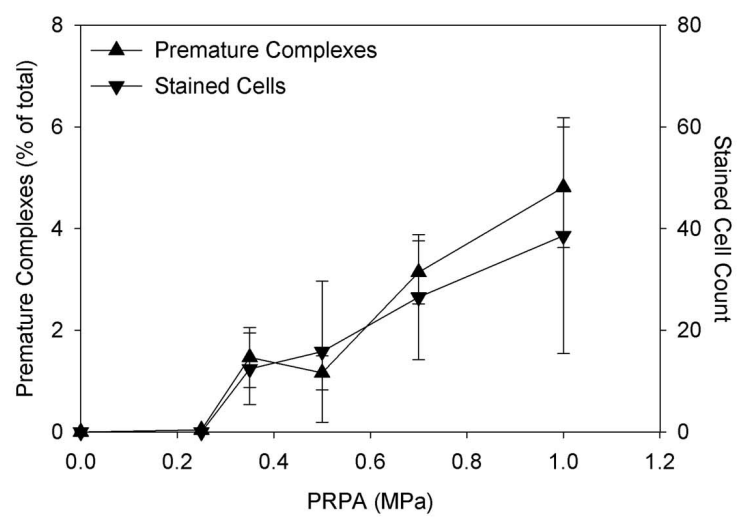

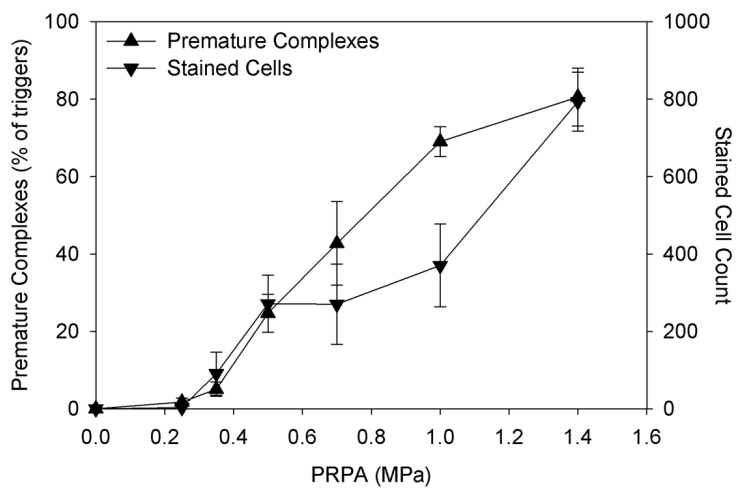

Figure 4. Means with SE bars for the numbers of PCS and Evans blue-stained SCs found for the triggered burst mode. The results were much greater than for the continuous burst mode (Figure 3), but again, the data trends with PRPAs are similar for both effects.

ECG, which allowed refill of blood containing contrast agent gas bodies, produced much larger counts of PCs and SCs. For the triggered single bursts, both effects were not statistically significant at $0.25 \mathrm{MPa}$ but were at $0.35 \mathrm{MPa}$, indicating a threshold of $0.3 \mathrm{MPa}$ for both bioeffects. For the triggered pulse envelope, both bioeffects were significant at 1.4 MPa but not at 1.0 $\mathrm{MPa}$, indicating thresholds of 1.2 MPa. Modes and thresholds are listed in Table 1.

The trends in the data for all 3 modes seem to be very similar for both the PCs and SCs. Results for the 2 effects were examined by linear regres-

Figure 5. Means with SE bars for the numbers of PCS and Evans blue-stained SCS found for the triggered pulse envelope mode. The results are diminished for the pulsed mode relative to the burst mode (Figure 4), but the data trends with PRPAs remain similar for both effects.

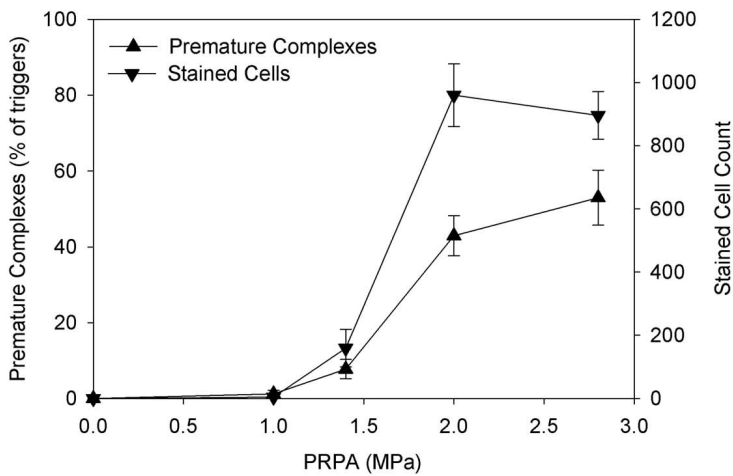


Table 1. Apparent Thresholds for PCs and SCs for the 3 Exposure Modes and Correlation Coefficients for Regression Against Individual Data and Means

\begin{tabular}{llllll}
\hline \multirow{2}{*}{ Mode } & \multicolumn{2}{l}{ Threshold, MPa } & & \multicolumn{2}{c}{$\boldsymbol{r}^{2}$} \\
\cline { 2 - 3 } \cline { 5 - 6 } & PC & SC & & Data & Mean \\
\hline Continuous burst & 0.3 & $0.85^{\mathrm{a}}$ & & 0.01 & 0.87 \\
Triggered single bursts & 0.3 & 0.3 & & 0.72 & 0.85 \\
Triggered pulse envelope & 1.2 & 1.2 & 0.85 & 0.96 \\
\hline
\end{tabular}

Apparent thresholds are defined as the midpoint between the lowest megapascal level with a significant effect and the next lower level. aThe SC effect was not statistically significant below 1.0 MPa, but SCs were observed in 3 of 5 rat heart samples at $0.35 \mathrm{MPa}, 2$ of 5 at 0.5 $\mathrm{MPa}$, and 3 of 4 at $0.7 \mathrm{MPa}$.

sion on the individual data and the means. Results for the 3 modes are shown in Figures 6-8 with $r^{2}$ values listed in Table 1. Regression for the continuous burst mode showed no correlation between the 2 effects for individual data, which was highly variable, but good correlation for the means. For the triggered single burst and pulse envelope modes, the correlation was good for both the individual data and the means. The results for triggered exposure strengthen the association between these 2 bioeffects.

The results for the ramp envelope exposure are shown in Figure 9. The ramp-up and ramp-down envelopes produced a different relative occurrence of the 2 bioeffects. The ramp-up condition produced slightly fewer, but not significantly fewer, PCs than the ramp-down condition. The SC counts were reduced significantly $(P<.001)$ for the ramp-up relative to the ramp-down condition.

Figure 6. Linear regressions of the SC and PC counts for the continuous burst mode. Although the data means (squares, bidirectional error bars, and dashed line) have a good correlation, the individual data points (circles and solid line) do not (Table 1).

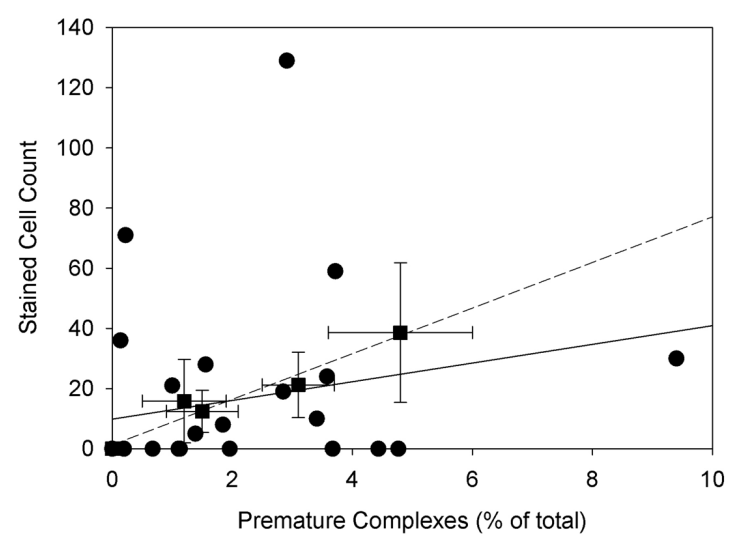

\section{Discussion}

Premature complexes in the ECG record have been reported for ultrasound with a gas body contrast agent for diagnostic B-mode ultrasound above about 1.0 MPa and also for the burst mode at substantially lower PRPAs. For B-mode ultrasound, injured cardiomyocytes have been associated with PCs. The objective of this study was to determine whether the PCs seen with burst mode exposure at lower PRPAs are also associated with cardiomyocyte injury. Three modes were used for characterizing the 2 bioeffects versus the exposure PRPA: a continuous burst (2 milliseconds on, 6 milliseconds off), single bursts, and pulse envelopes both triggered at 1:4 heartbeats during end systole. All 3 modes produced similar trends for both bioeffects, which was a steady increase with PRPAs above an apparent threshold (Figures 3-5). The continuous burst mode produced somewhat variable results, for which the 2 bioeffects were not correlated (except for regression on the means). It seems likely that the variability resulted from the continuous nature of the exposure, which might have prevented refilling of the capillary bed with blood containing gas bodies. The bioeffects thresholds were both $0.3 \mathrm{MPa}$ for the triggered burst mode and both 1.2 MPa for the square pulse envelope (Table 1). The pulse envelope thresholds were similar to those found for diagnostic imaging exposure. ${ }^{7}$ For burst mode exposure, irreversible cardiomyocyte injury was induced together with

Figure 7. Linear regressions of the SC and PC counts for the triggered burst mode. Both the data means (squares, bidirectional error bars, and dashed line) and the individual points (circles and solid line) have a good correlation (Table 1).

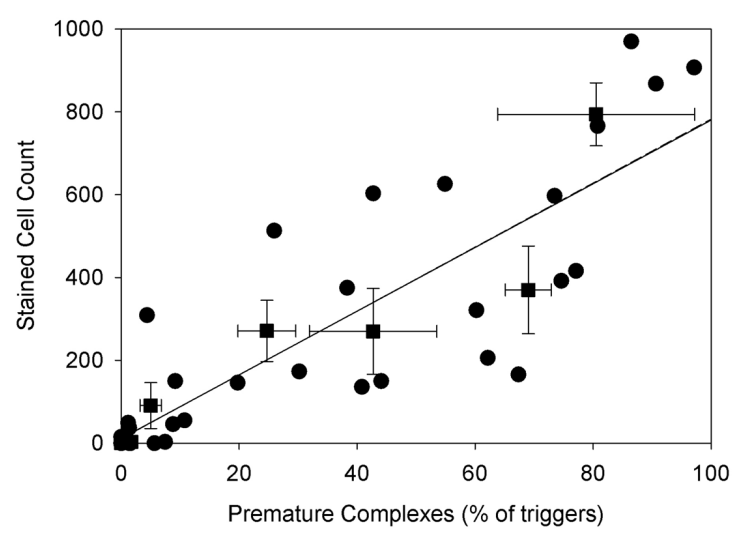




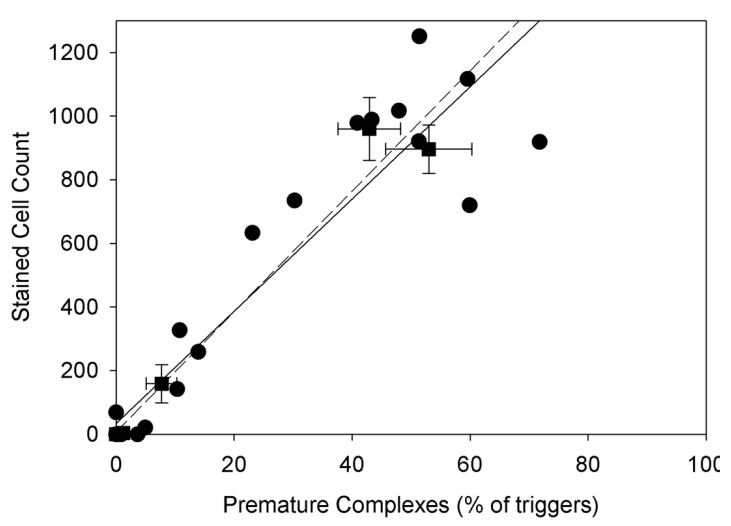

Figure 8. Linear regressions of the SC and PC counts for the triggered pulse envelope mode. Both the data means (squares, bidirectional error bars, and dashed line) and the individual points (circles and solid line) have a good correlation (Table 1).

arrhythmia at substantially lower PRPAs than for pulsed ultrasound. There was a good correlation, especially for the triggered modes, between the 2 bioeffects (Figures 6-8 and Table 1).

For tests with a ramped pulse envelope, the ramp-up envelope reduced the cardiomyocyte injury $(P<.001)$, but the arrhythmia bioeffect was not statistically different from that for the ramp-down condition. In previous work, the ramp-up envelope essentially abolished the glomerular capillary hemorrhage effect in rat kidneys, which was seen with the ramp-down envelope. ${ }^{12}$ This mitigation of the capillary hemorrhage was thought to be due to the destabilization and loss of potentially effective gas bodies

Figure 9. Comparison of the SC and PC counts for the ramped pulse envelopes. The numbers of PCs are not significantly different for the 2 modes, but the numbers of SCS are lower $(\mathrm{P}<$ .001) for the ramp up (increasing pulse PRPA).

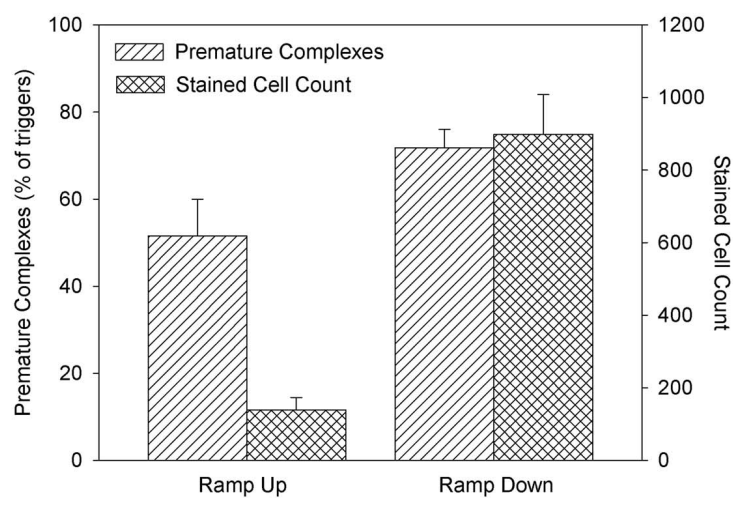

J Ultrasound Med 2009; 28:1519-1526 by relatively low-amplitude pulses in the ramp up, before the suprathreshold pulses arrived (for the ramp down, the reverse is true). For the cardiac bioeffects observed in this study, the rampup envelope did not abolish either bioeffect, and SCs were associated with the occurrence of PCs even for this bioeffects mitigation mode.

The 2 bioeffects, PCs and injured cardiomyocytes, induced by intermittent cardiac exposure to ultrasound with a gas body contrast agent occurred together, with similar thresholds, exposureresponse trends, and a good correlation for the lowPRPA burst mode as well higher-amplitude pulsed ultrasound. This shows that both bioeffects are caused by gas body activation, with destabilization and nucleation of cavitation, at similar PRPAs. Irreversible cardiomyocyte injury may be expected whenever PCs develop from intracardiac ultrasonic cavitation activity.

\section{References}

1. Miller DL, Averkiou MA, Brayman AA, et al. Bioeffects considerations for diagnostic ultrasound contrast agents. J Ultrasound Med 2008; 27:611-632.

2. van Der Wouw PA, Brauns AC, Bailey SE, Powers JE, Wilde $A A$. Premature ventricular contractions during triggered imaging with ultrasound contrast. J Am Soc Echocardiogr 2000; 13:288-294.

3. Chapman S, Windle J, Xie F, McGrain A, Porter TR. Incidence of cardiac arrhythmias with therapeutic versus diagnostic ultrasound and intravenous microbubbles. J Ultrasound Med 2005; 24:1099-1107.

4. Li P, Cao LQ, Dou CY, Armstrong WR, Miller DL. Impact of myocardial contrast echocardiography on vascular permeability: an in vivo dose response study of delivery mode, ultrasound power and contrast dose. Ultrasound Med Biol 2003; 29:1341-1349.

5. Miller DL, Driscoll EM, Dou C, Armstrong WF, Lucchesi BR. Microvascular permeabilization and cardiomyocyte injury provoked by myocardial contrast echocardiography in a canine model. J Am Coll Cardiol 2006; 47:1464-1468.

6. Miller DL, Li P, Gordon D, Armstrong WF. Histological characterization of microlesions induced by myocardial contrast echocardiography. Echocardiography 2005; 22:25-34.

7. Miller DL, Li P, Dou C, Gordon D, Edwards CA, Armstrong WF. Influence of contrast agent dose and ultrasound exposure on cardiomyocyte injury induced by myocardial contrast echocardiography in rats. Radiology 2005; 237: 137-143.

8. Zachary JF, Hartleben SA, Frizzell LA, O'Brien WD Jr. Arrhythmias in rat hearts exposed to pulsed ultrasound after intravenous injection of a contrast agent. J Ultrasound Med 2002; 21:1347-1356. 


\section{Ultrasound-Induced Cardiac Arrhythmia and Injury}

9. Carstensen EL, Gracewski S, Dalecki D. The search for cavitation in vivo. Ultrasound Med Biol 2000; 26:1377-1385.

10. Porter TR, Everbach C, Kricsfeld D, Xie F. Myocardial cavitational activity during continuous infusion and bolus intravenous injections of perfluorocarbon-containing microbubbles. J Am Soc Echocardiogr 2001; 14:618-625.

11. Hamlin RL. Non-drug-related electrocardiographic features in animal models in safety pharmacology. J Pharmacol Toxicol Methods 2005; 52:60-76.

12. Miller DL, Dou C, Wiggins RC. Simulation of diagnostic ultrasound image pulse sequences in cavitation bioeffects research. J Acoust Soc Am 2007; 122:2002-2008. 